\title{
WIGNER CRYSTAL STATE FOR THE EDGE ELECTRONS \\ IN THE QUANTUM HALL EFFECT AT FILLING $\nu=2$
}

\author{
J. P. Rodriguez, ${ }^{*}$ M.J. Franco ${ }^{\dagger}$ and L. Brey \\ Instituto de Ciencia de Materiales de Madrid, Consejo Superior de \\ Investigaciones Científicas, Cantoblanco, 28049 Madrid, Spain
}

\begin{abstract}
The electronic excitations at the edges of a Hall bar not much wider than a few magnetic lengths are studied theoretically at filling $\nu=2$. Both mean-field theory and Luttinger liquid theory techniques are employed for the case of a null Zeeman energy splitting. The first calculation yields a stable spin-density wave state along the bar, while the second one predicts dominant Wigner-crystal correlations along the edges of the bar. We propose an antiferromagnetic Wigner-crystal groundstate for the edge electrons that reconciles the two results. A net Zeeman splitting is found to produce canting of the antiferromagnetic order.
\end{abstract}

PACS Indices: $73.40 . \mathrm{Hm}, 72.15 . \mathrm{Nj}$

\footnotetext{
* Permanent address: Dept. of Physics and Astronomy, California State University, Los Angeles, CA 90032, USA.

$\dagger$ Present address: Meta4 Human Technology, Centro Europa Empresarial - Edf. Roma, 28230 Las Rozas, Madrid, Spain.
} 


\section{Introduction}

It was conjectured long ago by Wigner that a zero-temperature gas of electrons should transit into a crystalline state as the electron density is lowered to sufficiently dilute levels. ${ }^{1}$ The conjecture is based on the observation that the electronic wavefunctions are localized when kinetic energy is absent from the quantum mechanical Hamiltonian. This corresponds to the former dilute limit. ${ }^{2}$ More recently, Schulz studied the electron gas confined to one dimension through a bosonization analysis. ${ }^{3}$ He found that the zero-temperature groundstate is best described by a Wigner crystal state at all densities. The complete absence of a Luttinger liquid state, ${ }^{4}$ which is the paradigm for one-dimensional (1D) Fermi systems that experience short-range interactions, is consistent with the incomplete screening that is characteristic of the electron gas confined to a $1 \mathrm{D}$ geometry.

Wigner crystals have also been proposed as groundstates of quantum Hall effect systems at sufficiently low two-dimensional (2D) electron densities. ${ }^{5}$ Recently, two of the authors have shown that the auto-correlations for $2 k_{F}$ charge-density wave (CDW) order along the edges of a thin quantum Hall bar at electronic filling $\nu=1$ decay slower than any power law of the separation. ${ }^{6}$ The groundstate is fully spin polarized in such case. The same dependence was obtained by Schulz for the Wigner crystal $\left(4 k_{F}\right)$ auto-correlations in the case of the electron gas confined to one dimension in zero field. ${ }^{3}$ It stands to reason then that the groundstate of the edge-electrons for a thin quantum Hall bar at filling $\nu=1$ is effectively a spin-polarized 1D electron gas with dominant Wigner-crystal correlations.

In this paper, we shall study the nature of the groundstate for the edge electrons of a thin quantum Hall bar at filling $\nu=2,{ }^{7}$ in which case both the spin up and the spin down lowest Landau levels are completely filled. Our main conclusion is that such edge electrons are best described by a 1D Wigner crystal state with quasi long-range antiferromagnetic order for Hall bars of widths not much larger than a few magnetic lengths. Evidence in support of this claim has two sources. First, we find that a spin-density wave (SDW) state for the edge-electrons is favored over a CDW one in the absence of Zeeman energy splitting at the mean-field level. (A CDW groundstate for the edge-electrons was obtained within the mean-field approximation for the case of filling $\nu=1$ in ref. 6.) The amplitude of

the SDW is exponentially small for Hall bars of widths much larger than a few magnetic lengths, however. ${ }^{8}$ This calculation is presented in the next section. Second, we obtain 
dominant Wigner-crystal $4 k_{F}$ correlations that decay slower than any power law in the absence of Zeeman energy splitting from a standard bosonization analysis of the Luttinger model for the edge electrons. ${ }^{6-8}$ Similar results were obtained by Schulz for the unpolarized $1 \mathrm{D}$ electron gas. ${ }^{3}$ This is the subject of section III of the paper. The two results just cited for the groundstate of the edge-electrons of a quantum Hall bar at filling $\nu=2$ appear at first sight to be in conflict with each other. To bring them into accord, we then propose in section IV that the groundstate for the edge electrons is a $1 \mathrm{D}$ antiferromagnetic Mott insulator. ${ }^{2}$ The previous restriction to zero Zeeman energy splitting is relaxed at this stage, where the remaining spin- $1 / 2$ degrees of freedom are described by an effective antiferromagnetic chain in external magnetic field. This yields a canted 1D Neel state for the ground-state spin configuration. We find that the spin auto-correlations of this model compare favorably with the SDW autocorrelations obtained from the bosonization analysis of the Luttinger model at null Zeeman splitting (see section III). This lends theoretical support to our proposal.

\section{Mean-field Theory: CDW versus SDW}

As is well known, the quantum Hall effect is a physical realization of the two-dimensional (2D) electron liquid in strong magnetic field. ${ }^{9}$ The one-electron states are confined to the lowest Landau level in such case. Consider henceforth the open geometry particular to a Hall bar of length $L_{y}$ and of width $W$. We shall also assume periodic boundary conditions along both the $x$ (width) and the $y$ (length) directions, with each of the respective periods, $L_{x}$ and $L_{y}$, being much greater than $W$. The quantum mechanical description of this system is therefore given by the Hamiltonian $H=H_{0}+H_{1}$, with kinetic energy

$$
H_{0}=\sum_{x, s} \varepsilon_{x, s} c_{x, s}^{\dagger} c_{x, s}
$$

and with Coulomb interaction energy

$$
H_{1}=\left(2 L_{x} L_{y}\right)^{-1} \sum_{s_{1}, s_{2}} \sum_{x_{1}, x_{2}, \vec{q}} V(\vec{q}) e^{i q_{x}\left(x_{1}-x_{2}-q_{y} l_{0}^{2}\right)} e^{-q^{2} l_{0}^{2} / 2} c_{x_{1}, s_{1}}^{\dagger} c_{x_{2}, s_{2}}^{\dagger} c_{x_{2}+q_{y} l_{0}^{2}, s_{2}} c_{x_{1}-q_{y} l_{0}^{2}, s_{1}}
$$

expressed in second quantized form. ${ }^{6}$ In particular, the operator $c_{x, s}^{\dagger}$ creates a spin $s=\uparrow, \downarrow$ electron in the lowest Landau level at transverse position $x$ within the Hall bar. We 
take the Landau gauge, which means that the longitudinal momentum of this eigenstate along the bar is equal to $k_{y}=x / l_{0}^{2}$, where $l_{0}=(\hbar c / e B)^{1 / 2}$ is the magnetic length. The corresponding one-electron eigenenergy has the form

$$
\varepsilon_{x, s}=\varepsilon_{0}(x)+s E_{Z} / 2, \quad(s=+,-)
$$

where $E_{Z}=g_{0} \mu_{B} H$ is the Zeeman energy splitting of the lowest Landau level. (The equivalent quantum numbers $x$ and $k_{y}$ will be freely interchanged throughout the paper.) The dispersion, $\varepsilon_{0}(x)$, of the Landau levels is dictated by the nature of the edge potentials (see Fig. 1 and ref. 10). Last, $V(q)=2 \pi e^{2} / \epsilon q$ above represents the Fourier transform of the repulsive Coulomb interaction, where $\epsilon$ denotes the dielectric constant. Depending on the 2D electron density, $n$, the lowest Landau level can be (fractionally) filled by a factor of $\nu=2 \pi l_{0}^{2} n$. The $\nu=2$ state, for example, consists of completely filled lowest Landau levels for both of the spin- $\frac{1}{2}$ quantum numbers. Below, we present evidence for the existence of dominant SDW correlations along the edges of the Hall bar at this filling through a mean-field calculation.

Two of the authors have found theoretical evidence for a CDW instability of the edge electrons in the quantum Hall state at $\nu=1,{ }^{6}$ in which case the lowest Landau level corresponding to spin up is completely filled, while the spin down level is empty. We shall now determine the nature of such Peierls instabilities in the case where both of the lowest Landau levels are filled $(\nu=2)$. In order to simplify the analysis that follows, we shall first assume a null Zeeman energy splitting, $E_{Z}=0$. This constraint will be relaxed later in section IV. Consider now the CDW/SDW trial wavefunction

$$
\left|D W_{n}\right\rangle=\Pi_{k, s}\left[u_{k}^{\prime}+s^{n} v_{k}^{\prime} c_{k+g(k), s}^{\dagger} c_{k-g(k), s}\right] \cdot \Pi_{|q| \leq k_{F}, s} c_{q, s}^{\dagger}|0\rangle
$$

with coherence factors $u_{k}^{\prime}$ and $v_{k}^{\prime}$, and with index $n=0,1$. Here, $g(k)=(\operatorname{sgn} k) k_{F}$ denotes one-half of the CDW/SDW nesting vector. Its magnitude coincides with the Fermi wavenumber, $k_{F}=\frac{1}{2} W / l_{0}^{2}$, with respect to the momentum quantum number of the one-electron states parallel to the edges of the Hall bar. In the trivial case that $u_{k}^{\prime}=1$ and $v_{k}^{\prime}=0$, we then have a Fermi sea of free electron states localized at transverse position $x$ that extends out to the (Fermi) edges of the Hall bar: i.e., $|x|<\frac{1}{2} W$ (see Fig. 1). In the more general case $v_{k}^{\prime} \neq 0$, however, $\left|D W_{0}\right\rangle$ represents a CDW state, with nesting vector 
$2 k_{F}$, while $\left|D W_{1}\right\rangle$ represents the corresponding SDW state with staggered magnetization oriented parallel to the spin quantization axis $\hat{z}$. Note that the absence of Zeeman energy splitting implies that the Hamiltonian $H_{0}+H_{1}$ has $S U(2)$ spin rotation symmetry, which guarantees that SDW states formed along any other quantization axis are degenerate. (It will be shown in section IV that the presence of a nonzero Zeeman energy splitting favors transverse SDW states.) The corresponding oscillations in the electronic density and in the electronic spin that characterize these states are computed at the end of Appendix A. The CDW state, for example, shows $\cos \left(2 k_{F} y\right)$ oscillations of the electron density at longitudinal position $y$ along the Hall bar, while the SDW state instead shows such oscillations in the spin density of the edge electrons [see Eq. (A16)]. Such oscillations are found to be exponentially small, however, for Hall bars of widths that are much greater than a few magnetic lengths [see Eq. (A17) and ref. 8].

To proceed further, it is useful to observe that the groundstate wavefunction (4) can be re-expressed as the product,

$$
\left|D W_{n}\right\rangle=\Pi_{k, s} A_{k, s}^{\dagger}|0\rangle
$$

of quasiparticle excitations

$$
A_{k, s}^{\dagger}=u_{k} c_{k-k_{F}, s}^{\dagger}+s^{n} v_{k} c_{k+k_{F}, s}^{\dagger}
$$

with the restriction to wave-numbers $|k|<k_{F}$. This restriction shall be implicit in all subsequent sums and products over quantum numbers. Above, we have the identities $u_{k}=u_{k}^{\prime}$ and $v_{k}=v_{k}^{\prime}$ for $k>0$, and $u_{k}=v_{k}^{\prime}$ and $v_{k}=u_{k}^{\prime}$ for $k<0$. The normalization condition $\left\langle D W_{n} \mid D W_{n}\right\rangle=1$ thus imposes the standard constraint

$$
\left|u_{k}\right|^{2}+\left|v_{k}\right|^{2}=1
$$

on the coherence factors. Notice that the quasiparticle creation operators then also satisfy the normal fermionic commutation relations

$$
\left\{A_{i}, A_{j}^{\dagger}\right\}=\delta_{i, j}
$$

and

$$
\left\{A_{i}, A_{j}\right\}=0=\left\{A_{i}^{\dagger}, A_{j}^{\dagger}\right\}
$$


where $i=(k, s)$ and $j=\left(k^{\prime}, s^{\prime}\right)$ enumerate the corresponding quantum numbers. We can now make use of the identity (5) in conjunction with the above commutation relations to compute the groundstate energy of the trial density wave state (4). The kinetic energy $E_{0}=\left\langle D W_{n}\left|H_{0}\right| D W_{n}\right\rangle$ for the CDW/SDW state is thereby obtained and found to take the form

$$
E_{0}=2 \sum_{x} \varepsilon_{0}(x) n_{x}
$$

Here

$$
n_{x}=\left|u_{x+k_{F}}\right|^{2}+\left|v_{x-k_{F}}\right|^{2}
$$

is the mean quasiparticle occupation.

The calculation of the Coulomb energy $E_{1}=\left\langle D W_{n}\left|H_{1}\right| D W_{n}\right\rangle$ requires more work, however. First, it is useful to note that this expectation value is equal to the sum, $E_{1}=$ $E_{\text {dir }}+E_{\text {exc }}$, of a direct Coulomb energy

$$
E_{\text {dir }}=\sum_{s_{1}, s_{2}} \sum_{x_{1}, x_{2}, q} B\left(q, x_{1}-x_{2}\right)\left\langle c_{x_{1}, s_{1}}^{\dagger} c_{x_{1}-q, s_{1}}\right\rangle\left\langle c_{x_{2}, s_{2}}^{\dagger} c_{x_{2}+q, s_{2}}\right\rangle
$$

and of an exchange Coulomb energy

$$
E_{\mathrm{exc}}=-\sum_{s_{1}, s_{2}} \sum_{x_{1}, x_{2}, q} B\left(q, x_{1}-x_{2}\right)\left\langle c_{x_{1}, s_{1}}^{\dagger} c_{x_{2}+q, s_{2}}\right\rangle\left\langle c_{x_{2}, s_{2}}^{\dagger} c_{x_{1}-q, s_{1}}\right\rangle .
$$

Here, we define the effective potential

$$
B(q, x)=\left(2 L_{x} L_{y}\right)^{-1} \sum_{k} V(k, q) e^{i k(x-q)} e^{-\left(k^{2}+q^{2}\right) / 2} .
$$

Above, and hence-forth, we measure all lengths in units of the magnetic length: $l_{0} \rightarrow 1$. After assuming the form (5) for the CDW/SDW groundstate wavefunction, it is demonstrated in the Appendix A that the corresponding particle-hole expectation values that appear above are given by

$$
\left\langle c_{x, s}^{\dagger} c_{x^{\prime}, s^{\prime}}\right\rangle=\delta_{s, s^{\prime}} \sum_{w, w^{\prime}= \pm}(w)_{x, s}^{*}\left(w^{\prime}\right)_{x^{\prime}, s^{\prime}} \delta_{x, x^{\prime}+k_{F}}\left(w-w^{\prime}\right),
$$

with the coherence factors renamed $(-)_{x, s}=u_{x+k_{F}}$ and $(+)_{x, s}=s^{n} v_{x-k_{F}}$ for convenience [see Appendix A, Eqs. (A1)-(A7)]. The product $e_{\text {dir }}=\left\langle c_{x_{1}, s_{1}}^{\dagger} c_{x_{1}-q, s_{1}}\right\rangle \cdot\left\langle c_{x_{2}, s_{2}}^{\dagger} c_{x_{2}+q, s_{2}}\right\rangle$ that appears in expression (12) for the direct Coulomb energy thus takes the form

$$
e_{\mathrm{dir}}=\sum_{w, w^{\prime}= \pm}(w)_{x_{1}, s_{1}}^{*}\left(w^{\prime}\right)_{x_{1}-q, s_{1}} \delta_{q, k_{F}}\left(w-w^{\prime}\right) \cdot \sum_{z, z^{\prime}= \pm}(z)_{x_{2}, s_{2}}^{*}\left(z^{\prime}\right)_{x_{2}+q, s_{2}} \delta_{q, k_{F}\left(z^{\prime}-z\right)} .
$$


On the other hand, the product $e_{\mathrm{exc}}=\left\langle c_{x_{1}, s_{1}}^{\dagger} c_{x_{2}+q, s_{2}}\right\rangle \cdot\left\langle c_{x_{2}, s_{2}}^{\dagger} c_{x_{1}-q, s_{1}}\right\rangle$ that appears in expression (13) for the exchange Coulomb energy can be obtained from the direct term (16) through the replacement $q \rightarrow-q+x_{1}-x_{2}$ in the case of like spins, $s_{1}=s_{2}$ [see Appendix A, Eq. (A12)]. It is zero otherwise. After some manipulation [see Appendix A, Eqs. (A11) and (A13)], we therefore obtain the final form

$$
E_{1}=\sum_{x_{1}, x_{2}} D_{x_{1}, x_{2}} n_{x_{1}} n_{x_{2}}+\sum_{x_{1}, x_{2}} D_{x_{1}, x_{2}}^{\prime} \operatorname{Re}\left(u_{x_{1}}^{*} v_{x_{1}} u_{x_{2}} v_{x_{2}}^{*}\right)
$$

for the total Coulomb interaction energy, with direct and exchange kernals

$$
\begin{aligned}
D_{x_{1}, x_{2}} & =4 B\left(0, x_{1}-x_{2}\right)-2 B\left(x_{1}-x_{2}, x_{1}-x_{2}\right), \\
D_{x_{1}, x_{2}}^{\prime}=\delta_{n, 0} \cdot 4 \sum_{w= \pm} B\left(2 k_{F} w, x_{1}-x_{2}+2 k_{F} w\right) & \\
& -2 \sum_{w= \pm} B\left(x_{1}-x_{2}, x_{1}-x_{2}+2 k_{F} w\right),
\end{aligned}
$$

respectively. Observe that the exchange kernal (19) diverges logarithmically at the Fermi surface, $x_{1}=0=x_{2}$, with an attractive sign. The divergent nature of the Peierls instability present in the 1D Fermi sea $\left(v_{k}^{\prime}=0\right)$ then implies that the edge-electrons are unstable to CDW/SDW condensates (4). Yet the difference in energy between the CDW $(n=0)$ and SDW $(n=1)$ states $(4)$ is then equal to

$$
E_{\mathrm{CDW}}-E_{\mathrm{SDW}}=4 \sum_{x_{1}, x_{2}} \sum_{w= \pm} B\left(2 k_{F} w, x_{1}-x_{2}+2 k_{F} w\right) \cdot \operatorname{Re}\left(u_{x_{1}}^{*} v_{x_{1}} u_{x_{2}} v_{x_{2}}^{*}\right)
$$

for a given set of coherence factors. The sum

$$
\sum_{w= \pm} B\left(2 k_{F} w, x_{1}-x_{2}+2 k_{F} w\right)=2 B\left(2 k_{F}, x_{1}-x_{2}+2 k_{F}\right)
$$

is positive, however, which yields the inequality

$$
E_{\mathrm{SDW}}<E_{\mathrm{CDW}}
$$

If we now take the coherence factors to be those that minimize the CDW energy functional $E_{\mathrm{CDW}}\left[u_{k}, v_{k}\right]$, then the above inequality necessarily implies that $\min E_{\mathrm{SDW}}<\min E_{\mathrm{CDW}}$. We conclude, therefore, that the SDW state is more stable than the CDW states within the present mean-field theory approximation. 


\section{Luttinger Liquid Model}

The previous meanfield result indicates that SDW correlations along the edges of a thin Hall bar are likely to be important at filling $\nu=2$ in the absence of Zeeman splitting, $E_{Z}=0$. Recent work by two of the authors indicates that the groundstate of the edge

electrons in the fully spin-polarized $\nu=1$ state is a $\mathrm{CDW},{ }^{6}$ which is consistent with this result. These authors found, however, that the long-range CDW autocorrelations decay slower than any power law, contrary to what occurs in the case of short-range interactions. ${ }^{4}$ In addition, Schulz showed that the 1D electron gas is better described by a Wigner crystal state than by a CDW or SDW one. ${ }^{3}$ Could a Wigner crystal state then be the true electronic groundstate at the edges of the quantum Hall bar in the absence of Zeeman splitting $\left(E_{Z}=0\right)$ for filling $\nu=2$ as well? Indeed, we shall demonstrate below that the low-energy long-wavelength effective theory for the edge-electrons in such case corresponds to that of a modified 1D electron gas.

In order to answer the question just posed, we need to obtain more information about the dominant long-wavelength electronic correlations at the edges of the Hall bar for filling $\nu=2$. To this end, we introduce the following components of the Luther-Emery (LE) model Hamiltonian, ${ }^{11} H=H_{\|}+H_{\perp}$, that can describe the low-energy dynamics of the edge electrons at zero temperature in the absence of Zeeman splitting:

$$
\begin{aligned}
H_{\|}=\sum_{s=\uparrow, \downarrow} \int d y\left[v_{F}\left(L_{s}^{\dagger} i \partial_{y} L_{s}-R_{s}^{\dagger} i \partial_{y} R_{s}\right)\right. & +g_{2, \|} L_{s}^{\dagger} R_{s}^{\dagger} R_{s} L_{s}+ \\
& \left.+g_{4, \|}\left(L_{s}^{\dagger} L_{s} L_{s}^{\dagger} L_{s}+R_{s}^{\dagger} R_{s} R_{s}^{\dagger} R_{s}\right)\right], \\
H_{\perp}=\int d y\left[g_{1, \perp}\left(L_{\uparrow}^{\dagger} R_{\downarrow}^{\dagger} L_{\downarrow} R_{\uparrow}+\text { h.c. }\right)\right. & \left.+g_{2, \perp}\left(L_{\uparrow}^{\dagger} L_{\uparrow} R_{\downarrow}^{\dagger} R_{\downarrow}+R_{\uparrow}^{\dagger} R_{\uparrow} L_{\downarrow}^{\dagger} L_{\downarrow}\right)\right]+ \\
& +g_{4, \perp}\left(L_{\uparrow}^{\dagger} L_{\uparrow} L_{\downarrow}^{\dagger} L_{\downarrow}+R_{\uparrow}^{\dagger} R_{\uparrow} R_{\downarrow}^{\dagger} R_{\downarrow}\right) .
\end{aligned}
$$

Here, $e^{i k_{F} y} R_{s}(y)=L_{y}^{-1 / 2} \sum_{k} e^{i k y} a_{s}(k)$ and $e^{-i k_{F} y} L_{s}(y)=L_{y}^{-1 / 2} \sum_{k} e^{i k y} b_{s}(k)$ denote field operators for right and left moving spin $s$ fermions along the respective edges of the Hall bar. In particular, $a_{s}(k)$ and $b_{s}(k)$ denote the respective annihilation operators for 
spin $s$ edge electrons of momentum $k$ that move along the right and the left edges of the Hall bar, with a Fermi surface at $\pm k_{F}$. Note that point splitting and normal ordering of the interaction terms in $H_{\|}$and in $H_{\perp}$ is implicit where necessary. ${ }^{4}$ Spin-charge separation then yields the factorization $H_{\|}+H_{\perp}=H_{\rho}+H_{\sigma}$ of the electronic excitations along the coupled edges of the Hall bar, where

$$
\begin{aligned}
H_{\rho} & =\sum_{q>0} \sum_{j=R, L} 2 \pi v_{\rho}(q) \rho_{j}(q) \rho_{j}(-q)+\sum_{q} g_{\rho}(q) \rho_{R}(q) \rho_{L}(-q) \\
H_{\sigma} & =\sum_{q>0} \sum_{j=R, L} 2 \pi v_{\sigma}(q) \sigma_{j}(q) \sigma_{j}(-q)+\sum_{q} g_{\sigma}(q) \sigma_{R}(q) \sigma_{L}(-q)+H_{\perp, 1}
\end{aligned}
$$

are the respective commuting portions of the Hamiltonian. Here,

$$
\rho_{j}(q)=2^{-1 / 2}\left[\rho_{j}(q, \uparrow)+\rho_{j}(q, \downarrow)\right] \quad \text { and } \quad \sigma_{j}(q)=2^{-1 / 2}\left[\rho_{j}(q, \uparrow)-\rho_{j}(q, \downarrow)\right]
$$

are the standard particle-hole operators for collective charge and spin excitations, with $\rho_{R}(q, s)=L_{y}^{-1 / 2} \sum_{k} a_{s}^{\dagger}(q+k) a_{s}(k)$ and $\rho_{L}(q, s)=L_{y}^{-1 / 2} \sum_{k} b_{s}^{\dagger}(q+k) b_{s}(k)$, while $H_{\perp, 1}=$ $g_{1, \perp} \sum_{s} \int d y L_{s}^{\dagger} R_{-s}^{\dagger} L_{-s} R_{s}$ is the LE spin-backscattering process. Also, the Fermi velocities and interaction strengths for each component are renormalized by the forward scattering processes [see Eqs. (22) and (23)] to

$$
\begin{aligned}
& v_{\rho, \sigma}=v_{F}+\left(g_{4, \|} \pm g_{4, \perp}\right) / 2 \pi, \\
& g_{\rho, \sigma}=g_{2, \|} \pm g_{2, \perp},
\end{aligned}
$$

where the $+(-)$ signs above correspond to the $\rho(\sigma)$ label. Last, it is important to remark that $S U(2)$ spin rotation invariance yields the identity

$$
g_{2, \|}-g_{2, \perp}=-g_{1, \perp}
$$

between $g_{\sigma}=g_{2, \|}-g_{2, \perp}$ and the strength of the spin-backscattering interaction, in addition to the identity

$$
g_{4, \|}=g_{4, \perp}
$$

between the forward scattering processes.

Let us now determine the interaction parameters that are theoretically expected for the above LE model in the case of the quantum Hall bar with null Zeeman splitting. ${ }^{7}$ Since 
the Coulomb interaction is spin independent, we have the identities

$$
\begin{aligned}
& g_{2, \|}=\frac{1}{2} V_{\text {inter }}=g_{2, \perp} \\
& g_{4, \|}=\frac{1}{2} V_{\text {intra }}=g_{4, \perp}
\end{aligned}
$$

between the above LE model interaction parameters and the Fourier transforms

$$
\begin{aligned}
& V_{\text {intra }}(q)=\frac{2 e^{2}}{\epsilon} \ln \frac{\gamma_{0}}{q W} \\
& V_{\text {inter }}(q)=\frac{2 e^{2}}{\epsilon} K_{0}(q W)
\end{aligned}
$$

of the respective intra-edge and the inter-edge Coulomb potentials. Above, $\gamma_{0}$ is a constant of order unity and $K_{0}(x)$ is a modified Bessel function. These assignments then yield a wave-number dependent Luttinger liquid for the charge sector (compare with ref. 6), parametrized by

$$
v_{\rho}(q)=v_{F}+V_{\text {intra }}(q) / 2 \pi \quad \text { and } \quad g_{\rho}(q)=V_{\text {inter }}(q),
$$

and a free theory,

$$
v_{\sigma}=v_{F} \quad \text { and } \quad g_{\sigma}=0=g_{1, \perp}
$$

for the spin sector. The latter necessarily implies that the spin gap is null. As a first probe of the nature of this system, we shall compute the static one-particle correlation function at asymptotically large separations via the standard abelian bosonization technique. ${ }^{12}$ Spincharge separation yields the form $\left\langle\Psi_{s}(0) \Psi_{s}^{\dagger}(y)\right\rangle=G_{\rho}(y) G_{\sigma}(y)$ for this quantity, where

$$
\Psi_{s}(y)=L_{s}(y)+R_{s}(y)
$$

is the field operator that coherently removes a spin $s$ edge electron at longitudinal position $y$ along the Hall bar. The bosonization technique yields the expressions $G_{\rho}(y) \sim$ $\exp \left[-\right.$ const. $\left.\times(\ln y)^{3 / 2} /(\ln W)^{1 / 2}\right]$ and $G_{\sigma}(y) \sim\left(\alpha_{0} / y\right)^{1 / 2}$ for the asymptotic one-particle autocorrelators of the charge and spin sectors, respectively. Here, $\alpha_{0}$ denotes the natural ultra-violet length scale of the edge electrons. The non-algebraic dependence of $G_{\rho}(y)$ is a result of the wave-number dependence (34) of the Luttinger model parameters due to the Coulomb interaction (see refs. 3 and 6 ). We therefore obtain the asymptotic result

$$
\left\langle\Psi_{s}(0) \Psi_{s}^{\dagger}(y)\right\rangle \sim\left(\alpha_{0} / y\right)^{1 / 2} \exp \left[- \text { const. } \times(\ln y)^{3 / 2} /(\ln W)^{1 / 2}\right]
$$


for the static one-particle autocorrelator, which notably decays faster than any power law. This clearly indicates that the edge electrons of the quantum Hall bar at $\nu=2$ are not in a Luttinger liquid state in the absence of Zeeman splitting, $E_{Z}=0$. [It should be remarked that the both the spin and the charge sectors of the LE model, Eqs. (24) and (25), exhibit Lorentz invariance. This implies that dynamic autocorrelations are related to the corresponding static ones by a suitable identification of the time difference $t$ with the relative displacement $y$. (See refs. 7, 8 and 12.)]

To gain yet more information about the nature of the correlated state along the edges of the $\nu=2$ quantum Hall bar, we shall now compute the CDW and SDW auto-correlation functions. Again, the bosonization technique shall be employed. The free spin sector (35) implies that the former are identical $\left(K_{\sigma}=1\right.$, see ref. 4). It is therefore sufficient to compute the autocorrelator for the $2 k_{F}$ CDW order parameter, $O_{2}=R_{\uparrow}^{\dagger} L_{\uparrow}+R_{\downarrow}^{\dagger} L_{\downarrow}$. It has the form $\left\langle O_{2}(0) O_{2}^{\dagger}(y)\right\rangle=\cos \left(2 k_{F} y\right) C_{\rho}(y) C_{\sigma}(y)$, where ${ }^{6} C_{\rho}(y) \sim \exp [-$ const.' $\times(\ln y$. $\ln W)^{1 / 2}$ ] and ${ }^{4} C_{\sigma}(y) \sim \alpha_{0} / y$ are the respective charge and spin contributions. This yields the result

$$
\left\langle O_{2}(0) O_{2}^{\dagger}(y)\right\rangle \sim \cos \left(2 k_{F} y\right) \cdot\left(\alpha_{0} / y\right) \cdot \exp \left[- \text { const. }^{\prime} \times(\ln y \cdot \ln W)^{1 / 2}\right]
$$

for the CDW/SDW autocorrelator asymptotically, which predominantly decays as $1 / y$ (see also ref. 8). Finally, we compute the auto-correlation function for the $4 k_{F}$ Wigner-crystal order parameter, $O_{4}=R_{\uparrow}^{\dagger} R_{\downarrow}^{\dagger} L_{\downarrow} L_{\uparrow}$. Application of the bosonization technique yields the asymptotic form

$$
\left\langle O_{4}(0) O_{4}^{\dagger}(y)\right\rangle \sim \cos \left(4 k_{F} y\right) \cdot \exp \left[-2 \text { const.' } \times(\ln y \cdot \ln W)^{1 / 2}\right]
$$

which decays slower than any power law. ${ }^{3}$ Notice that sub-algebraic factors are common to both the CDW/SDW and to the $4 k_{F}$ autocorrelators, (38) and (39). We conclude that Wigner crystal correlations are dominant along the edges of the quantum Hall bar in the absence of Zeeman energy splitting at filling $\nu=2$.

\section{Antiferromagnetic Wigner Crystal Proposal}

The prior Luttinger model analysis for the dynamics of the edge electrons along a quantum Hall bar at filling $\nu=2$ in the absence of Zeeman energy splitting indicates that 
the corresponding groundstate is in fact a $1 \mathrm{D}$ Wigner crystal of periodicity $a=\pi / 2 k_{F}$. Although SDW correlations are also found to be quasi long range, they are subdominant. The meanfield analysis presented in section II found dominant SDW order with respect to CDW order, however. These results seem to be incompatible at first site. In principle, the meanfield result could simply be wrong. We believe this not to be the case, however, for the following reasons. It can be easily shown that a meanfield analysis ${ }^{13}$ of the Luther-Emery model, (22) and (23), in terms of CDW, SDW, and Cooper pair order parameters for the edge electrons recovers the same phase diagram that is obtained from a renormalizationgroup analysis of the equivalent Coulomb-gas ensemble. ${ }^{4,12}$ This indicates that the meanfield analysis made in section II for the entire quantum Hall bar is correct in so far as the phase stability is concerned.

We propose instead the following solution to the puzzle outlined above: The groundstate of the edge electrons along a quantum Hall bar at $\nu=2$ is effectively described by a spin-1/2 antiferromagnetic chain in external magnetic field, with lattice constant

$$
a=\pi l_{0}^{2} / W
$$

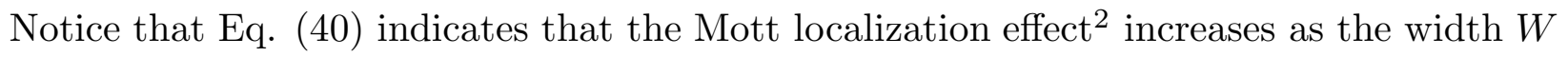
of the strip decreases. The dominant Wigner crystal correlations obtained in the previous section indicate that the effective 1D spin-1/2 fermions represented by the field operator $\Psi_{s}(y)$ [see Eq. (36)] are localized at longitudinal points $y_{i}=i \cdot a$ along the Hall bar. The remaining effective spin degree of freedom

$$
\vec{S}_{i}=\frac{1}{2} \sum_{s, s^{\prime}} \Psi_{s}^{\dagger}\left(y_{i}\right) \vec{\sigma}_{s, s^{\prime}} \Psi_{s^{\prime}}\left(y_{i}\right)
$$

is a coherent measure of the spin along both edges of the Hall bar. It is then governed by the Hamiltonian for the antiferromagnetic Heisenberg chain $(J>0)$ in external magnetic field $\vec{h}=E_{Z} \hat{z}:^{14}$

$$
H_{\sigma}=J \sum_{i} \vec{S}_{i} \cdot \vec{S}_{i+1}-\vec{h} \cdot \sum_{i} \vec{S}_{i}
$$

The spin-wave excitations of the chain also disperse linearly, ${ }^{15}$ with a group velocity $v_{\sigma}^{\prime}=$ $\pi J a / 2 \hbar$ in zero field. The latter is obtained from comparison with the exact Bethe ansatz solution for the spin-1/2 Heisenberg chain. ${ }^{16}$ Notice that the absence of a spin gap is 
consistent with the previous Luttinger model analysis. Indeed, comparison with Eq. (35) in turn yields the formula

$$
J=(2 / \pi)^{2} \hbar k_{F} v_{F}
$$

for the effective exchange-coupling constant in zero field.

The above spin-1/2 antiferromagnetic chain (42) is well understood theoretically. The groundstate is a Neel state (plus zero-point spin-wave fluctuations) that is canted in the direction of the external field. Indeed, the application of the Jordan-Wigner transformation to this spin chain yields an equivalent system of interacting $1 \mathrm{D}$ spinless fermions. ${ }^{17} \mathrm{~A}$ subsequent Luttinger model analysis then yields asymptotic transverse and longitudinal static spin correlations

$$
\begin{aligned}
\left\langle S_{i}^{+} S_{i+m}^{-}\right\rangle & \sim(-1)^{m} / m \\
\left\langle\delta S_{i}^{z} \delta S_{i+m}^{z}\right\rangle & \sim\left[\cos \left(2 k_{F} a \cdot m\right)\right] / m
\end{aligned}
$$

at low fields that are consistent with such a canted antiferromagnetic state (see Appendix B). Above, $\delta S_{z}$ refers to the deviation of the magnetization with respect to the mean spin polarization

$$
\left\langle S_{i}^{z}\right\rangle=\chi_{\perp} h_{z}
$$

along the direction $\hat{z}$ of the applied field, while $k_{F} \cong \pi / 2 a$ denotes the field-dependent Fermi wavenumber of the $1 \mathrm{D}$ spinless fermions. Here $\chi_{\perp}=1 / \pi^{2} J$ is the transverse magnetic susceptibility known exactly at zero field. ${ }^{16}$ In particular, the incommensurate SDW (45) represents the effect of canting, whereas the transverse spin correlations (44) describe the antiferromagnetic component of the configuration. Observe that the spin auto-correlators, (44) and (45), are quite similar to the SDW auto-correlator (38) obtain previously from the Luttinger model analysis for the edge-electron dynamics in the absence of Zeeman splitting. The only difference between the two results is the absence of an additional sub-algebraic factor in the spin auto-correlators for the antiferromagnetic chain. This is likely to be connected with the fact that the analog of the $4 k_{F}$ autocorrelator (39) in the spin-chain model (42) is simply the density-density correlation function, which is identically equal to unity, and which therefore shows no such sub-algebraic dependence. Last, the canted Neel state saturates to a fully polarized state at a critical field $h_{c} \sim J$. Comparison of the edge band structure depicted in Fig. 1 with Eq. (43) indicates, however, 
that the system has passed into a $\nu=1$ quantum Hall state by then. The later nevertheless possesses a ferromagnetic groundstate spin configuration and Wigner-crystal-type correlations along the edges. ${ }^{6}$ This coincides precisely with the nature of the saturated canted antiferromagnetic state at $h_{c}$.

Given the above Wigner-crystal proposal, we can now go back and make contact with the previous mean-field calculation presented in section II. In the limit of small Zeeman

field, $\vec{h} \rightarrow 0$, the change in energy of a SDW state in comparison to its zero-field value takes the bilinear form

$$
\delta E_{\mathrm{SDW}} / \mathcal{N}=-\frac{1}{2}\left(\chi_{\|} h_{\|}^{2}+\chi_{\perp} h_{\perp}^{2}\right)
$$

in terms of the parallel and perpendicular paramagnetic susceptibilities and fields with respect to the staggered magnetization of the SDW. Here $\mathcal{N}$ denotes the total number of conduction electrons in the Hall bar. The presence of a single-particle gap in the mean-field excitation spectrum implies that the longitudinal susceptibility is null, however, at zero temperature: $\chi_{\|}=0$. Also, comparison with the previous antiferromagnetic chain yields a non-zero transverse paramagnetic susceptibility, $\chi_{\perp} \sim J^{-1}$. Eq. (47) then indicates that the lowest-energy SDW states are those with the staggered magnetization oriented perpendicular to the external field: e.g.

$$
\left|S D W_{x}\right\rangle=\Pi_{k, s}\left[u_{k}^{\prime}+v_{k}^{\prime} c_{k+g(k), s}^{\dagger} c_{k-g(k),-s}\right] \cdot \Pi_{|q| \leq k_{F}, s} c_{q, s}^{\dagger}|0\rangle .
$$

Examination of Fig. 1 reveals that such mean-field SDW states permit a unique definition of the nesting vector, $2 k_{F}$. The free Fermi sea thus experiences a Peierls instability that mixes the spin quantum number following Fig. 1. Notice, however, that a unique nesting vector cannot be defined in either the case of the CDW or of the longitudinal SDW states, $\left|D W_{0}\right\rangle$ and $\left|D W_{1}\right\rangle=\left|S D W_{z}\right\rangle$, for $E_{Z}>0$. This is undoubtedly the principal reason why such states are not stable in general. [We remind the reader that the longitudinal (4) and transverse (48) SDW states are degenerate in the absence of Zeeman energy splitting due to $S U(2)$ spin rotation symmetry.]

\section{Conclusions}

By combining the results obtained from a mean-field theory and a Luttinger liquid analysis of the edge-electrons of a thin quantum Hall bar at filling $\nu=2$, we propose 
that the corresponding groundstate is in a Wigner crystal state, with the remaining spin degrees of freedom arranged into a canted antiferromagnetic configuration. The amplitude of both the respective CDW and SDW is exponentially small, however, for Hall bars of width much larger than a few magnetic lengths [see Appendix A, Eqs. (A16) and (A17), and ref. 8]. We remind the reader that two of the authors have shown that a similar Wigner crystal/CDW state is expected theoretically along the edges of a quantum Hall bar in the fully spin-polarized state found at filling $\nu=1 .^{6}$

There are various experimental tests that could identify the above Wigner-crystal canted antiferromagnetic state at $\nu=2$. First, it is known that any $1 \mathrm{D}$ Wigner crystal is pinned by a single "backscattering" impurity. ${ }^{18,19}$ In the present context, such a defect corresponds to a correlated pin that extends in the transverse direction from one edge of the Hall bar to the other. Nonlinear conduction along the length of the Hall bar is then expected below a threshold voltage, while ohmic conduction is expected above this threshold. The former corresponds to a pinned Wigner crystal state while the latter corresponds to a sliding Wigner crystal state. Second, a good probe for the predicted SDW order is the spin-lattice relaxation rate, $1 / T_{1}$, in nuclear magnetic resonance. ${ }^{8}$ This quantity is directly proportional to the transverse spin-correlator (44), which means that it is sensitive to the canted antiferromagnetic order predicted here theoretically.

J.P.R. thanks E. Rezayi for discussions. He also acknowledges partial support from the National Science Foundation through grant \# DMR-9322427, and partial support from the Spanish Ministry for Education and Culture. L.B. was supported in part by the Spanish Ministry for Education and Culture under contract \# PB 960085. 


\section{Appendix A: Evaluation of $\left\langle c_{x^{\prime}, s^{\prime}}^{\dagger} c_{x, s}\right\rangle$, etc.}

Eqs. (12) and (13) indicate that the essential object in the evaluation of the Coulomb energy (2) with respect to the CDW/SDW groundstate [Eqs. (4) and (5)] is the particlehole expectation value

$$
\left\langle D W_{n}\left|c_{x^{\prime}, s^{\prime}}^{\dagger} c_{x, s}\right| D W_{n}\right\rangle=\left\langle 0\left|\Pi_{i^{\prime}} A_{i^{\prime}} \cdot c_{x^{\prime}, s^{\prime}}^{\dagger} c_{x, s} \cdot \Pi_{i} A_{i}^{\dagger}\right| 0\right\rangle .
$$

The latter can be computed by progressively commuting the annihilation operator $c_{x, s}$ to the right, and by progressively commuting the creation operator $c_{x^{\prime}, s^{\prime}}^{\dagger}$ to the left above. This process results in the identity

$$
c_{x, s}\left|D W_{n}\right\rangle=\sum_{i}(-1)^{i+1}\left\{c_{x, s}, A_{i}^{\dagger}\right\} \Pi_{j \neq i} A_{j}^{\dagger}|0\rangle
$$

for the correlated hole excitation. Evidently, it is a coherent sum of all possible hole excitations weighted by the appropriate commutator

$$
\left\{c_{x, s}, A_{i}^{\dagger}\right\}=\delta_{s, s_{i}}\left(u_{k_{i}} \delta_{x, k_{i}-k_{F}}+s_{i}^{n} v_{k_{i}} \delta_{x, k_{i}+k_{F}}\right) .
$$

Use of the identity $\left\langle 0\left|A_{i} A_{j}^{\dagger}\right| 0\right\rangle=\delta_{i, j}$ for the quasiparticle excitations then yields the expression

$$
\left\langle D W_{n}\left|c_{x^{\prime}, s^{\prime}}^{\dagger} c_{x, s}\right| D W_{n}\right\rangle=\sum_{i}\left\{A_{i}, c_{x^{\prime}, s^{\prime}}^{\dagger}\right\}\left\{c_{x, s}, A_{i}^{\dagger}\right\}
$$

for the expectation value (A1). Finally, substitution of (A3) above yields the desired result

$$
\left\langle D W_{n}\left|c_{x^{\prime}, s^{\prime}}^{\dagger} c_{x, s}\right| D W_{n}\right\rangle=\delta_{s, s^{\prime}} \sum_{w, w^{\prime}= \pm}(w)_{x^{\prime}, s^{\prime}}^{*}\left(w^{\prime}\right)_{x, s} \delta_{x^{\prime}, x+k_{F}\left(w-w^{\prime}\right)}
$$

for the expectation value in terms of the coherence factors, which have been renamed as

$$
\begin{aligned}
& (-)_{x, s}=u_{x+k_{F}} \\
& (+)_{x, s}=s^{n} v_{x-k_{F}}
\end{aligned}
$$

for convenience.

We shall next evaluate the products

$$
\begin{aligned}
e_{\text {dir }} & =\left\langle D W_{n}\left|c_{x_{1}, s_{1}}^{\dagger} c_{x_{1}-q, s_{1}}\right| D W_{n}\right\rangle \cdot\left\langle D W_{n}\left|c_{x_{2}, s_{2}}^{\dagger} c_{x_{2}+q, s_{2}}\right| D W_{n}\right\rangle \\
e_{\text {exc }} & =\left\langle D W_{n}\left|c_{x_{1}, s_{1}}^{\dagger} c_{x_{2}+q, s_{2}}\right| D W_{n}\right\rangle \cdot\left\langle D W_{n}\left|c_{x_{2}, s_{2}}^{\dagger} c_{x_{1}-q, s_{1}}\right| D W_{n}\right\rangle
\end{aligned}
$$


that appear in expressions (12) and (13) for the direct and exchange contributions to the Coulomb interaction energy. Substitution of the prior result (A5) into (A8), for example, yields the expression

$$
e_{\mathrm{dir}}=\sum_{w, w^{\prime}= \pm}(w)_{x_{1}, s_{1}}^{*}\left(w^{\prime}\right)_{x_{1}-q, s_{1}} \delta_{q, k_{F}\left(w-w^{\prime}\right)} \cdot \sum_{z, z^{\prime}= \pm}(z)_{x_{2}, s_{2}}^{*}\left(z^{\prime}\right)_{x_{2}+q, s_{2}} \delta_{q, k_{F}\left(z^{\prime}-z\right)}
$$

for the direct term. After some manipulation, one obtains the final result

$$
\begin{aligned}
e_{\mathrm{dir}}=\delta_{q, 0} & \sum_{w= \pm}\left|(w)_{x_{1}, s_{1}}\right|^{2} \sum_{w^{\prime}= \pm}\left|\left(w^{\prime}\right)_{x_{2}, s_{2}}\right|^{2}+ \\
& +\sum_{w= \pm} \delta_{q, 2 k_{F} w}(w)_{x_{1}, s_{1}}^{*}(\bar{w})_{x_{1}-2 k_{F} w, s_{1}}(\bar{w})_{x_{2}, s_{2}}^{*}(w)_{x_{2}+2 k_{F} w, s_{2}}
\end{aligned}
$$

for the product (A8), where we define the notation $\bar{w}=-w$. In the case of the exchange term (A9), on the other hand, substitution of (A5) yields the expression

$$
\begin{aligned}
e_{\mathrm{exc}}=\delta_{s_{1}, s_{2}} \sum_{w, w^{\prime}= \pm}(w)_{x_{1}, s_{1}}^{*}\left(w^{\prime}\right)_{x_{2}+q, s_{2}} \delta_{x_{1}-x_{2}-q, k_{F}}\left(w-w^{\prime}\right) & \times \\
& \times \sum_{z, z^{\prime}= \pm}(z)_{x_{2}, s_{2}}^{*}\left(z^{\prime}\right)_{x_{1}-q, s_{1}} \delta_{x_{1}-x_{2}-q, k_{F}\left(z^{\prime}-z\right)}
\end{aligned}
$$

Again, after some manipulation, we arrive at the result

$$
\begin{aligned}
e_{\mathrm{exc}}=\delta_{s_{1}, s_{2}}\left(\delta_{q, x_{1}-x_{2}}\right. & \sum_{w= \pm}\left|(w)_{x_{1}, s_{1}}\right|^{2} \sum_{w^{\prime}= \pm}\left|\left(w^{\prime}\right)_{x_{2}, s_{2}}\right|^{2}+ \\
& \left.+\sum_{w= \pm} \delta_{q, x_{1}-x_{2}-2 k_{F} w}(w)_{x_{1}, s_{1}}^{*}(\bar{w})_{x_{1}-2 k_{F} w, s_{1}}(\bar{w})_{x_{2}, s_{2}}^{*}(w)_{x_{2}+2 k_{F} w, s_{2}}\right) .
\end{aligned}
$$

Notice that $e_{\text {exc }}$ can be obtained from $e_{\text {dir }}$ by making the replacement $q \rightarrow-q+x_{1}-x_{2}$ in the case of like spins, $s_{1}=s_{2}$ [see Eqs. (A8) and (A9)].

Last, we shall compute the expectation value of the number/spin density operator

$$
S^{(\mu)}(\vec{r})=\sum_{s, s^{\prime}} \sum_{k, k^{\prime}} a_{s, s^{\prime}}^{(\mu)}\langle k \mid \vec{r}\rangle\left\langle\vec{r} \mid k^{\prime}\right\rangle c_{k, s}^{\dagger} c_{k^{\prime}, s^{\prime}}
$$

at a point $\vec{r}=(x, y)$ in the Hall bar. Here $a^{(1)}, a^{(2)}$ and $a^{(3)}$ are the Pauli matrices corresponding to the spin density, while $a_{s, s^{\prime}}^{(0)}=\delta_{s, s^{\prime}}$ corresponds to the number density. 
Also, the wavefunction $\langle k \mid \vec{r}\rangle=L_{y}^{-1 / 2} e^{i k y} \phi_{k}(x)$ represents an electron with momentum $k$ along the right edge of the Hall bar in the Landau gauge, with the guiding center factor $\phi_{k}(x)=\pi^{-1 / 4} l_{0}^{-1 / 2} \exp \left[-\left(x-k l_{0}^{2}\right)^{2} / 2 l_{0}^{2}\right]$. Use of the result (A5) for the particle-hole amplitude yields the expression

$$
\left\langle S^{(\mu)}(\vec{r})\right\rangle=\sum_{s} \sum_{k, k^{\prime}} a_{s, s}^{(\mu)}\langle k \mid \vec{r}\rangle\left\langle\vec{r} \mid k^{\prime}\right\rangle \sum_{w, w^{\prime}= \pm}(w)_{k, s}^{*}\left(w^{\prime}\right)_{k^{\prime}, s} \delta_{k, k^{\prime}+k_{F}\left(w-w^{\prime}\right)}
$$

for the expectation value of the number/spin density (A14). After some manipulation, we obtain the final form

$$
\begin{aligned}
\left\langle S^{(\mu)}(\vec{r})\right\rangle= & L_{y}^{-1} \sum_{k, s} a_{s, s}^{(\mu)}\left[\left|\phi_{k-k_{F}}(x)\right|^{2}\left|u_{k}\right|^{2}+\left|\phi_{k+k_{F}}(x)\right|^{2}\left|v_{k}\right|^{2}\right]+ \\
& +2 \cos \left(2 k_{F} y+\phi\right) L_{y}^{-1} \sum_{k, s}\left[a_{s, s}^{(\mu)} \cdot s^{n}\right] \phi_{k+k_{F}}(x) \phi_{k-k_{F}}(x)\left|u_{k}^{*} v_{k}\right|
\end{aligned}
$$

where $\phi$ is the relative phase in between the coherence factors at $k=0$. Since $\sum_{s} a_{s, s}^{(0)} \cdot s^{n}=$ 2 and $\sum_{s} a_{s, s}^{(3)} \cdot s^{n}=0$ for the CDW state, $\left|D W_{0}\right\rangle$, and vice versa for the SDW state, $\left|D W_{1}\right\rangle$, we conclude that the CDW state shows $\cos \left(2 k_{F} y+\phi\right)$ oscillations in the electronic density along the Hall bar, while the SDW state shows such oscillations in the electronic spin density along the $z$-axis [see the contributions to the second term in Eq. (A16) near $k=0]$. The amplitude of such oscillations is limited by the product

$$
\phi_{k+k_{F}}(x) \phi_{k-k_{F}}(x)=\pi^{-1 / 2} l_{0}^{-1} e^{-\left[\left(x-k l_{0}^{2}\right) / l_{0}\right]^{2}-\left(k_{F} l_{0}\right)^{2}}
$$

of the guiding center wavefunctions at the opposing edges, which is appreciable for thin Hall bars, $W \lesssim 2 l_{0} \cdot{ }^{8}$

\section{Appendix B: $S=1 / 2$ Antiferromagnetic Chain in External Field}

We shall now reproduce the well-known bosonization analysis ${ }^{14}$ of the $s=1 / 2$ antiferromagnetic chain (42) for the purpose of showing that the groundstate in external field is in a canted Neel configuration. It is useful to consider first the more general case with anisotropy described by the Hamiltonian

$$
H_{\sigma}=\sum_{i}\left[\frac{1}{2} J_{x y}\left(S_{i}^{+} S_{i+1}^{-}+S_{i+1}^{+} S_{i}^{-}\right)+J_{z} S_{i}^{z} S_{i+1}^{z}-h_{z} S_{i}^{z}\right]
$$


for the $s=1 / 2$ XXZ chain in external magnetic field $h_{z}$ aligned parallel to the anisotropy axis. Application of the Jordan-Wigner transformation ${ }^{17}$

$$
S_{i}^{-}=f_{i} e^{i \pi N_{i}} \text { and } S_{i}^{+}=f_{i}^{\dagger} e^{-i \pi N_{i}}
$$

of the lowering and raising operators to spinless fermions, $f_{i}$, plus the string $N_{i}=\sum_{j<i} f_{j}^{\dagger} f_{j}$ yields the equivalent fermion representation

$$
H_{\sigma}=\sum_{i}\left[\frac{1}{2} J_{x y}\left(f_{i}^{\dagger} f_{i+1}+\text { h.c. }\right)+J_{z} n_{i} n_{i+1}-h_{z} n_{i}\right]
$$

of the spin Hamiltonian (B1). Above, we have used the identity

$$
S_{i}^{z}=n_{i}-\frac{1}{2}
$$

in between the spin operator along the anisotropy axis and the occupation number $n_{i}=$ $f_{i}^{\dagger} f_{i}$ for the spinless fermions. This system is a compressible Luttinger liquid for $J_{x y} \geq J_{z}$, which translates into a paramagnetic response

$$
\left\langle S_{i}^{z}\right\rangle=\chi_{\perp} h_{z}
$$

Comparison of the latter with Eq. (B4) then indicates that the system of spinless fermions (B3) is half filled at zero field, and off half filling at $h_{z} \neq 0$. Following Luther and Peschel, ${ }^{14}$ the correlation functions can be easily calculation via the bosonization technique, where one obtains the results

$$
\begin{aligned}
\left\langle S_{i}^{-} S_{i+x / a}^{+}\right\rangle & \sim(-1)^{x / a}\left(\frac{\alpha_{0}}{x}\right)^{(2 K)^{-1}}, \\
\left\langle\delta S_{i}^{z} \delta S_{i+x / a}^{z}\right\rangle & \sim \cos \left(2 k_{F} x\right)\left(\frac{\alpha_{0}}{x}\right)^{2 K}
\end{aligned}
$$

in the asymptotic limit $x \rightarrow \infty$. Above, $k_{F}$ denotes the field-dependent Fermi wavenumber, while $K$ denotes the characteristic Luttinger liquid parameter. Also, $\alpha_{0}^{-1} \sim a^{-1}$ is the momentum cutoff of the underlying Luttinger model, while $\delta S_{i}^{z}=S_{i}^{z}-\left\langle S_{i}^{z}\right\rangle$. Notice that the string factors (B2) remove any dependence of the oscillatory factor in the transverse spin autocorrelator (B6) with $k_{F}$. 
In the case of the Heisenberg chain, $J_{x y}=J_{z}$, the spin correlations are isotropic at zero magnetic field. This requires that $2 K=1=(2 K)^{-1}$ in such case. We therefore obtain the spin autocorrelations

$$
\begin{aligned}
\left\langle S_{i}^{-} S_{i+m}^{+}\right\rangle & \sim(-1)^{m} / m, \\
\left\langle\delta S_{i}^{z} \delta S_{i+m}^{z}\right\rangle & \sim\left[\cos \left(2 k_{F} a \cdot m\right)\right] / m
\end{aligned}
$$

in the low field limit, where $k_{F} \cong \pi / 2 a$. Study of Eqs. (B5), (B8) and (B9) then indicates that the groundstate of the $s=1 / 2$ antiferromagnetic Heisenberg chain in the presence of a weak external magnetic field is in a canted Neel configuration. 


\section{References}

1. E. Wigner, Phys. Rev. 46, 1002 (1934).

2. N.F. Mott, Metal-Insulator Transitions (Taylor and Francis, London, 1990).

3. H.J. Schulz, Phys. Rev. Lett. 71, 1864 (1993).

4. J. Voit, Rep. Prog. Phys. 58, 977 (1995).

5. H.E. Fertig in Perspectives in Quantum Hall Effects, edited by S. Das Sarma and A. Pinczuk (Wiley 1997).

6. M. Franco and L. Brey, Phys. Rev. Lett. 77, 1358 (1996).

7. H.C. Lee and S.-R. Eric Yang, Phys. Rev. B 56, 15529 (1997).

8. H.C. Lee and S.-R. Eric Yang, Phys. Rev. B 57, R4249 (1998).

9. The Quantum Hall Effect, edited by R.E. Prange and S.M. Girvin, (Springer-Verlag, 1990), 2nd ed..

10. B.I. Halperin, Phys. Rev. B 25, 2185 (1982); X.G. Wen, Int. J. Mod. Phys. B 8, 457 (1994).

11. A. Luther and V.J. Emery, Phys. Rev. Lett. 33, 589 (1974).

12. V.J. Emery, in Highly Conducting One-dimensional Solids, ed. by J.T. Devreese, R.P. Evrard and V.E. van Doren (Plenum Press, New York, 1979).

13. J.P. Rodriguez, Europhys. Lett. 39, 195 (1997); Europhys. Lett. 47, 745 (E) (1999); Phys. Rev. B 58, 944 (1998).

14. A. Luther and I. Peschel, Phys. Rev. B 12, 3908 (1975).

15. F.D.M. Haldane, Phys. Rev. Lett. 45, 1358 (1980).

16. R.J. Baxter, Ann. Phys. (N.Y.) 70, 193 (1972).

17. E. Fradkin, Field Theories of Condensed Matter Systems (Addison-Wesley, Redwood City, 1991), chap. 4.

18. C.L. Kane and M.P.A. Fisher, Phys. Rev. B 46, 15233 (1992).

19. L.I. Glazman, I.M. Ruzin and B.I. Shklovskii, Phys. Rev. B 45, 8454 (1992). 


\section{Figure Caption}

Fig. 1. Shown is the schematic band structure of the edge electrons along the quantum Hall bar at filling $\nu=2$. The horizontal arrows represent the unique nesting vector, $2 k_{F}$, for the transverse SDW states [see Eq. (48), for example]. 


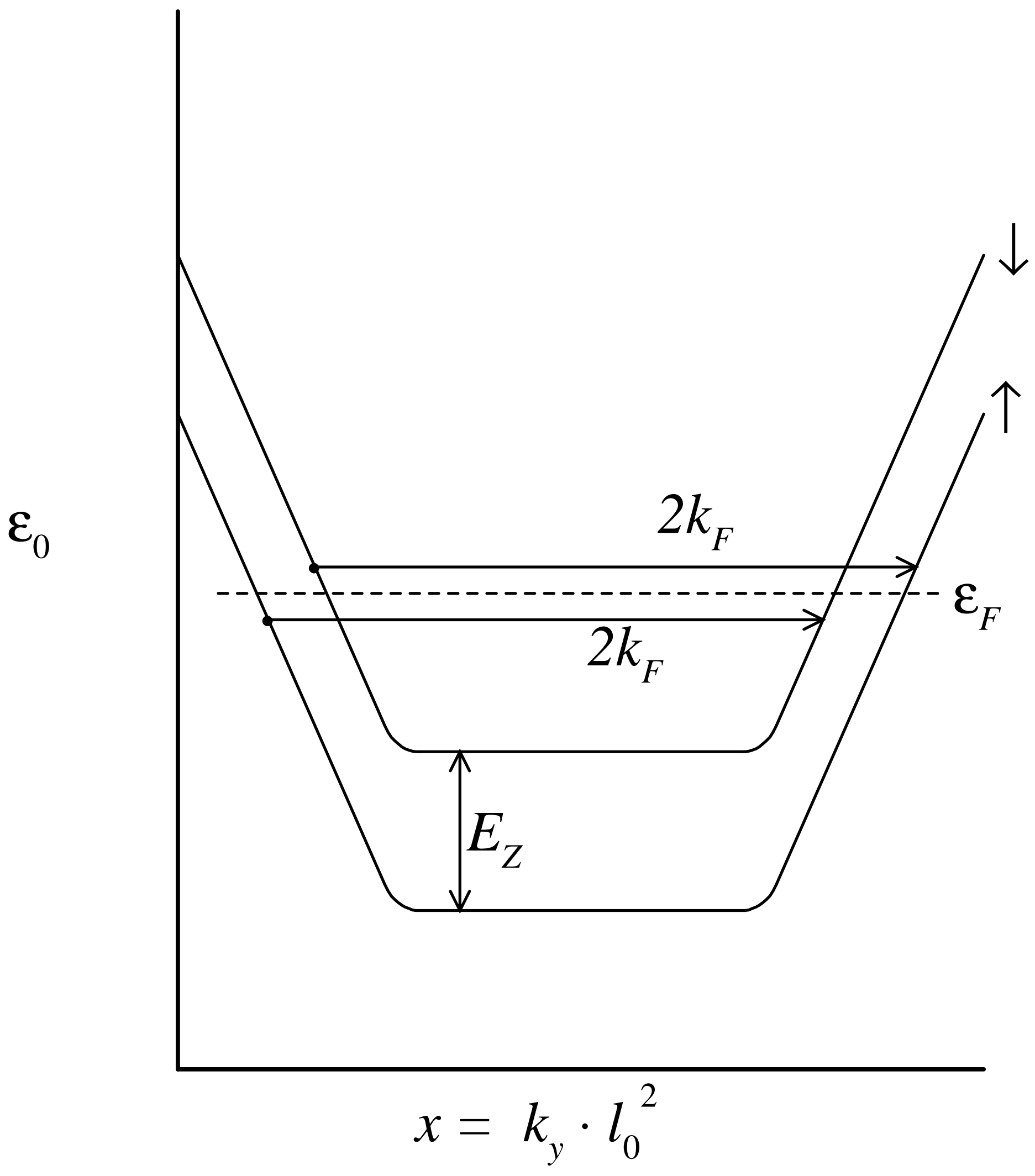

